\title{
Mid-Term Outcomes of Cemented Stem and Subtrochanteric Shortening Derotational Osteotomy in Total Hip Arthroplasty for Crowe IV Developmental Dysplasia
}

\author{
Zhe-Yu Huang \\ Ningbo No. 6 Hospital \\ Jing Ling \\ Ningbo No. 6 Hospital \\ Zhi-Min Zeng \\ Ningbo No. 6 Hospital \\ Zheng-Lin Di \\ Ningbo No. 6 Hospital \\ Jun-Hui Zhang \\ Ningbo No. 6 Hospital \\ Kun Tao ( $\square$ nb_joint@163.com ) \\ Ningbo No. 6 Hospital
}

\section{Research Article}

Keywords: subtrochanteric shortening derotational transverse osteotomy, cemented stem, Crowe IV developmental dysplasia of the hip, nonunion

Posted Date: August 4th, 2021

DOI: https://doi.org/10.21203/rs.3.rs-745383/v1

License: (c) (i) This work is licensed under a Creative Commons Attribution 4.0 International License. Read Full License 


\section{Abstract}

Background Performing total hip arthroplasty (THA) in patients with Crowe IV developmental dysplasia of the hip (DDH) is technically challenging. Subtrochanteric shortening osteotomy is typically required for placing the acetabular component within the anatomic hip center. However, the outcomes of subtrochanteric osteotomy using cemented components are not widely reported. This study aimed to evaluate the outcomes of cemented stem THA with subtrochanteric femoral shortening and transverse derotational osteotomy in patients with Crowe IV DDH.

Methods We retrospectively evaluated data of patients with Crowe IV DDH who underwent cemented stem THA with subtrochanteric femoral shortening and transverse derotational osteotomy between 2010 and 2018. Patients who underwent surgery at the hip joint were excluded. Data regarding pre- and postoperative clinical and radiological parameters were collected and reviewed.

Results Among 14 patients included (14 hips), the mean age was 60.4 (range, 47-73) years. The mean Harris hip score improved from 40.7 to 87.7 . The mean limb length discrepancy reduced from $52 \mathrm{~mm}$ to $12.7 \mathrm{~mm}$. No neurologic deficits were noted. The mean osteotomy union time was 10.6 months. Delayed union and postoperative dislocation were observed in one and two patients, respectively. Cement leakage into the osteotomy gap was observed in one patient. No revisions were required. No signs of loosening or migration were observed.

Conclusions Cemented stem THA combined with subtrochanteric femoral shortening and transverse derotational osteotomy is safe and effective for the treatment of patients with Crowe IV DDH. The cemented femoral component showed promising mid-term follow up results. However, cement leakage affects bone healing. Osteotomy and cementing should be performed meticulously.

Trial Registration: Retrospectively registered

\section{Background}

Total hip arthroplasty (THA) for Crowe IV developmental dysplasia of the hip (DDH) can be a very challenging procedure to perform, on both the femoral and acetabular sides, owing to the severe anatomical abnormalities associated with the disease. These abnormalities include low acetabular bone stock, abnormal hip center location, narrow femoral canal measurements, excessive femoral anteversion, valgus neck-shaft angles, posteriorly located greater trochanters, soft tissue contractures, large lower limb length discrepancies, and poor hip abductor mechanisms [1, 2]. One of the most important steps in THA is restoring the hip to the anatomical center of rotation, which corrects the functions of the abductor muscles $[3,4]$, resulting in long-term benefits. However, this step is associated with limb lengthening exceeding $40 \mathrm{~mm}$, thus increasing the risk for neurologic traction injury $[5,6]$. Subtrochanteric femoral shortening and derotational osteotomy were introduced to address these limitations. Subtrochanteric femoral shortening osteotomy helps to pull down the femur, correct the rotational abnormalities, preserve 
the proximal femoral metaphysis, and reduce the risk for nerve injury [7], while derotational osteotomy corrects the excessive femoral anteversion and posterior positioning of greater trochanter [8].

THA can be performed with cemented or uncemented stems. Subtrochanteric femoral shortening osteotomy with uncemented THA has been extensively studied. In contrast, the data on subtrochanteric femoral shortening osteotomy with cemented stems are lacking [8, 13-17]. Furthermore, four subtrochanteric osteotomy techniques are currently available. These techniques differ with respect to the osteotomy cutting shape, which may be transverse, oblique, double-chevron, or Z-shaped [9-12]. Among these, the most common is the transverse osteotomy because it is technically simple and allows the surgeon to adjust the anteversion angle and length of the osteotomy with ease $[18,19]$.

For these reasons, in the present study we elected to focus on the cemented stem THA with subtrochanteric femoral shortening transverse osteotomy in patients with Crowe IV DDH. We aimed to present the mid-term follow-up results and focused especially on the bone union status at the site of the femoral osteotomy.

\section{Methods}

\section{Clinical Data and Radiological Analysis}

This retrospective study protocol was approved by the appropriate Institutional Review Board (L2021023). Written informed consent was obtained from all patients.

We identified all patients with Crowe IV DDH who underwent subtrochanteric femoral shortening derotational transverse osteotomy between June 2015 and October 2018. The cemented Lubinus SP II femoral component (Waldemar Link, Hamburg, Germany) and cementless CombiCup acetabular component (Waldemar Link, Hamburg, Germany) were used in all cases. Patients who underwent surgery at the hip joint were excluded. Pre- and postoperative data, including clinical, operative, and radiological findings, as well as complications, were collected.

Clinical follow-up was conducted at 4 weeks, 12 weeks, and 6 months after surgery and annually thereafter until the last follow-up, left to the discretion of the attending surgeon. Patients were evaluated based on their Harris hip score (HHS), preoperative and postoperative limb length discrepancy (LLD), severity of limp, and Trendelenburg test results. LLD was calculated by measuring the distance between the anterior superior iliac spine and the medial malleolus.

Serial radiographs of the pelvis were used for radiographic analysis by two coauthors. Any discrepancies in findings were discussed to reach consensus. On the pelvic side, acetabular component loosening was diagnosed if any of the following were observed: a progressive radiolucent line with a thickness of more than $2 \mathrm{~mm}$ around the inserted cup, migration of the component, or a change in the cup position [20]. On the femoral side, we investigated the evolution of radiolucent lines in the seven femoral zones [21]. Loosening was defined according to the criteria described by Johnston et al. [22]. 
Limb lengthening was calculated using pre- and postoperative radiographs by subtracting the length of the resected femur from the distance between the tip of the greater trochanter [23]. Bone union at the osteotomy site was assessed using postoperative radiographs based on the criteria for bone union described by Masonis et al. [8]. The level of the osteotomy site was measured according to a report published in 2020 [24]. The length of the cemented stem bridging the osteotomy site was also noted.

\section{Surgical Technique}

All operations were performed by a senior author via a posterolateral approach with the patient in the lateral decubitus position. The sciatic nerve was initially identified to avoid iatrogenic injury. The joint capsule, femoral head, osteophytes, and any fibrous scar tissue were excised carefully and completely. After identifying the true acetabular floor and the transverse ligament, which are the landmarks for the center of the hip, the acetabulum was gradually thinned with hemispherical reamers until bleeding cancellous bone was visualized, indicating that the medial wall of the true acetabulum had been reached. If the acetabular bone was insufficient, a femoral head and neck bone autograft was used to enlarge and reinforce the acetabular roof. The acetabular component (CombiCup, Waldemar Link, Hamburg, Germany) was then inserted into the anatomical acetabular position via the press-fit technique and fixed with dome screws. The outer diameter of the acetabular cup was $44-50 \mathrm{~mm}$.

The femoral trial component was implanted at the level of the lesser trochanter. The need for subtrochanteri osteotomy was determined based on the preoperative plan and the intraoperative assessment of soft tissue tension while attempting the reduction of the femoral head into the acetabulum. This was tested by pulling the leg with the knee flexed at approximately $90^{\circ}$ while persistent distal traction was applied. If the femoral head was not reduced into the acetabulum, the distance between the top of the acetabular implant and the femoral head need to be measured. According to Huang et al. [24], initial osteotomy was performed at an optimal level below the lesser trochanter (the mean level of the initial osteotomy site was $21.1 \mathrm{~mm}$ [range, 15-30 mm]). The trial stem was then inserted into the proximal fragment, and the trial head was reduced into the cup. Manual traction was applied to the distal femur with the knee flexed at approximately $90^{\circ}$. The length of the bony overlap at the osteotomy level was measured, and this length was excised from the distal femur. The distal shaft of the femur should be broached again to account for the total length of the resected bone. The femoral trial component was then reinserted, which transfixed the osteotomy site. Reduction was then performed. Sciatic nerve tension was reassessed by palpating the nerve with the knee flexed at approximately $20^{\circ}$. The LLD, range of movement, and impingement were also assessed.

Next, the hip was prepared for the cemented implant. The contact faces of the proximal and distal cortical bones were planarized and three to five 3-mm holes were drilled near the osteotomy sites of both fragments. Both femoral fragments were held in place by bone clamps with the great trochanter towards outside, then the cement was applied. The final component was inserted into the proximal femur, with the implant anteverted at $10-15^{\circ}$. Ceramic-on-ceramic wear-bearing material was used in all patients. Patients were started immediately on partial weight-bearing exercises lasting approximately 8 weeks. If 
osseous healing was noted at the osteotomy site at 8-12 weeks, the patients gradually progressed to full weight-bearing exercises.

\section{Statistical Analysis}

Data are presented as mean values with ranges or mean \pm standard deviation. The Student's $t$-test was used to analyze continuous variables. Analyses of the correlation between the osteotomy union time and the length of femoral resection, length of limb lengthening, distance of the osteotomy site from the lesser trochanter, and length of the femoral stem bridging the osteotomy site were performed using Spearman's rank correlation coefficient. A $P$ value $<0.05$ was considered statistically significant. Statistical analyses were performed using SPSS software version 24.0 (IBM Corp., Armonk, NY, USA).

\section{Results}

Among 14 patients with Crowe IV DDH who underwent THA using a cemented Lubinus SP II stem with subtrochanteric femoral shortening and transverse derotational osteotomy between 2010 and 2018 ( $\mathrm{n}=$ 14 hips), there were 10 females and four males with a mean body mass index of 24.4 (range, 20.5-29.3) and 26.2 (range, 24.3-28.1), respectively, and a mean age of 60.4 years (range, 47-73 years) at the time of the operation. The mean follow-up period was 49.1 months (range, 30-70 months) (Table 1). No patient was lost to follow-up. The mean HHS improved significantly from 40.7 preoperatively to 87.7 postoperatively, and the mean LLD was significantly reduced from $52 \mathrm{~mm}$ to $12.7 \mathrm{~mm}$. No postoperative neurologic deficits were noted. One patient had bilateral Crowe IV DDH. No obvious leg length discrepancies were noted preoperatively; however, obvious LLDs were noted postoperatively. Preoperative Trendelenburg tests were positive for all patients, but postoperative Trendelenburg tests were negative for all patients at the last follow-up. A slight postoperative limp was noted in two patients, and no limp was noted in 12 patients (Table 2). The mean osteotomy union time was 10.6 months (range, 7.6-18 months). One patient experienced delayed union at 18 months postoperatively. In this case, radiographic examination revealed that some cement had leaked into the osteotomy gap (Fig. 1). 
Table 1

Patient Demographics and Outcome Measures

\begin{tabular}{|c|c|c|c|c|c|c|c|c|c|}
\hline \multirow{3}{*}{$\begin{array}{l}\text { Cases } \\
\\
1\end{array}$} & \multirow{3}{*}{$\begin{array}{l}\text { Age } \\
\text { (Years) } \\
50\end{array}$} & \multirow{3}{*}{$\begin{array}{l}\text { Gender } \\
\text { Female }\end{array}$} & \multirow{3}{*}{$\begin{array}{l}\text { BMI } \\
\\
25\end{array}$} & \multirow{3}{*}{$\begin{array}{l}\text { Follow } \\
\text { up } \\
\text { (Months) } \\
70\end{array}$} & \multirow{3}{*}{$\begin{array}{l}\text { Side } \\
\text { Right }\end{array}$} & \multirow{2}{*}{\multicolumn{2}{|c|}{$\begin{array}{l}\begin{array}{l}\text { Harris Hip } \\
\text { Score }\end{array} \\
\text { Preoperative } \\
\text { Last }\end{array}$}} & \multirow{3}{*}{ 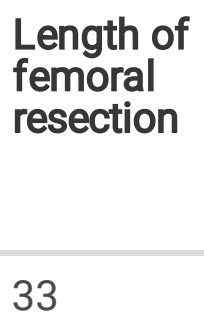 } & \multirow{3}{*}{$\begin{array}{l}\begin{array}{l}\text { Osteotomy } \\
\text { union time }\end{array} \\
9.9\end{array}$} \\
\hline & & & & & & & & & \\
\hline & & & & & & 38 & 88 & & \\
\hline 2 & 47 & Female & 20.5 & 66 & Right & 36 & 86 & 40 & 11.1 \\
\hline 3 & 68 & Male & 28.1 & 53 & Right & 40 & 84 & 38 & 11.8 \\
\hline 4 & 73 & Female & 23 & 45 & Right & 42 & 89 & 45 & 7.6 \\
\hline 5 & 51 & Female & 24.4 & 43 & Right & 37 & 93 & 35 & 18.0 \\
\hline 6 & 62 & Male & 24.3 & 38 & Right & 44 & 91 & 38 & 10.4 \\
\hline 7 & 72 & Female & 29.3 & 30 & Right & 48 & 83 & 40 & 11.1 \\
\hline 8 & 52 & Male & 26.4 & 55 & Left & 37 & 86 & 35 & 10.6 \\
\hline 9 & 65 & Female & 25.9 & 63 & Left & 38 & 87 & 43 & 8.2 \\
\hline 10 & 59 & Female & 25.2 & 30 & Right & 40 & 89 & 35 & 11.7 \\
\hline 11 & 66 & Male & 26 & 46 & Left & 40 & 87 & 40 & 11.5 \\
\hline 12 & 51 & Female & 22.7 & 65 & Left & 45 & 92 & 35 & 8.5 \\
\hline 13 & 62 & Female & 25 & 38 & Left & 40 & 90 & 40 & 8.5 \\
\hline 14 & 68 & Female & 23.1 & 45 & Right & 45 & 83 & 41 & 9.1 \\
\hline
\end{tabular}

Table 2

Clinical Results 


\begin{tabular}{|c|c|c|c|}
\hline Outcomes & Preoperative & Last Follow-Up & $p$ Value $^{\star}$ \\
\hline Harris Hip Score & & & $<.01$ \\
\hline Mean (range) & $40.7(36-48)$ & $87.7(83-93)$ & \\
\hline \multicolumn{4}{|l|}{ Limp (no. of hips) } \\
\hline Severe & 9 & 0 & \\
\hline Moderate & 3 & 0 & \\
\hline Slight & 2 & 2 & \\
\hline None & 0 & 12 & \\
\hline $\begin{array}{l}\text { Limb length discrepancy } \\
\text { (mm) (no. of hips) }\end{array}$ & & & $<.01$ \\
\hline$<10$ & 1 & 9 & \\
\hline $10-20$ & 0 & 4 & \\
\hline$>20$ & 13 & 1 & \\
\hline \multicolumn{4}{|l|}{$\begin{array}{l}\text { Trendelenburg sign } \\
\text { (no. of hips) }\end{array}$} \\
\hline Positive & 14 & 0 & \\
\hline Negative & 0 & 14 & \\
\hline * Student's t-test & & & \\
\hline
\end{tabular}

The mean length of the excised femoral segment was $38.4 \mathrm{~mm}$ (range, 33-45 mm), and the mean length of limb lengthening was $27.1 \mathrm{~mm}$ (range, 16-38 mm). We also measured the distance between the osteotomy site and lesser trochanter. The mean distance was $21.1 \mathrm{~mm}$ (range, 15-30 mm). The mean length of the femoral stem bridging the osteotomy site was $97.6 \mathrm{~mm}$ (range, 76.5-106.2 mm). No statistically significant correlation was found between the osteotomy union time and these factors (Table $3)$.

Table 3

Correlation of several factors with the osteotomy union time 


\begin{tabular}{|lll|}
\hline & Mean \pm SD & $p$ value* \\
\hline Osteotomy union time & $10.6 \pm 2.6$ & \\
\hline Length of femoral resection & $38.4 \pm 3.5$ & .097 \\
\hline Limb lengthening & $27.1 \pm 7.9$ & .323 \\
\hline Level of osteotomy site & $21.1 \pm 5.0$ & .595 \\
\hline Length of stem to bridge osteotomy site & $97.6 \pm 8.6$ & .309 \\
\hline * Spearman's rank correlation coefficient & & \\
\hline
\end{tabular}

Two patients experienced hip dislocation within 3 months of the index surgery (Fig. 2). Both patients underwent closed reduction and had no recurrence of hip dislocation at the last follow-up. No cases of infection, nerve palsy, or intraoperative fractures were noted during the study. No implant loosening was noted on radiographic analysis. No revision was performed for any reason.

\section{Discussion}

In the present study, we presented the mid-term follow-up data focused on the bone union status at the site of the femoral osteotomy in patients who underwent cemented stem THA with subtrochanteric femoral shortening transverse osteotomy for Crowe IV DDH. Our results indicate that this procedure is safe and effective based on clinical and radiological outcomes.

Performing THA in patients with Crowe IV DDH is an effective but difficult procedure. To improve the prosthesis survival rate and reduce limb length discrepancies, proper positioning of the acetabular component within the real acetabulum is necessary [25]. However, this procedure may cause the pullingdown distance of the hip center to exceed $40 \mathrm{~mm}$, which can jeopardize neurovascular structures $[5,6]$. Subtrochanteric femoral shortening and derotational osteotomy were introduced to address this limitation, but they were also associated with a higher risk for other complications, particularly nonunion at the osteotomy site. Nonunion of the osteotomy site may lead to varus angulation, pain, loss of rotational stability, and prosthetic loosening $[7,26]$. The proper union of the osteotomy site depends on several factors, such as the stability of the osteotomy site [27], size of the contact area between the proximal and distal segments [24], available bone stock, and degree of vascularization. Among these factors, the stability of the osteotomy site is the most important and is contingent on the osteotomy type, stem fixation method, stem morphology, and use of additional fixation materials such as cables or plate and screws $[26,28]$. However, the impact on osteotomy site stability associated with the osteotomy type and stem fixation method remains contested in the literature.

As a technique, subtrochanteric osteotomy is very adaptable and can be performed with transverse, oblique, double-chevron, and Z-shaped osteotomy shapes [9-12]. Transverse osteotomy reportedly has lower rotational stability than other techniques [7, 27]. However, Muratli et al. [27] conducted a biomechanical study that compared the four osteotomy shapes and demonstrated that no single inherent 
feature increased osteotomy site stability. In addition, a comprehensive meta-analysis by Li et al. [18] reported no significant differences in complications and survival rates according to the four cutting shapes. However, transverse osteotomy is the preferred approach because it is a relatively simple technique that allows surgeons to adjust the anteversion angle with minimal damage to the periosteum at the osteotomy site $[18,19]$.

Many surgeons also prefer performing subtrochanteric osteotomy with an uncemented femoral stem [912] because cemented stems are prone to failure from cracking or fatigue owing to a narrow cement mantle [29]. Aseptic loosening has also been reported in young and middle-aged patients who received cemented stems [30]. Moreover, cemented stems perform worse in revision surgeries because of the inadequate residual bone stock [31]. They are also associated with a risk for cement leakage into the osteotomy gap [32], which can lead to nonunion of the osteotomy site. Some studies have reported that cemented THA with subtrochanteric osteotomy is an effective treatment for severely dislocated hips [8, $13-17]$. However, the nonunion rate with cemented prostheses is $0-20 \%[8,13-17]$ compared with $0-$ $12.5 \%$ with uncemented prostheses $[1,33,34]$. The nonunion rate was similar between cemented and cementless stems. Recent improvements in prosthesis materials, product manufacturing processes, and cementing techniques have led to better results with cemented stems [35]. In the clinical setting, the main concern is that the cement will leak into the osteotomy site, leading to nonunion. However, Kawai et al. [36] reported that cemented THA with subtrochanteric transverse osteotomy provides satisfactory shortterm results with no major complications in patients with Crowe IV DDH. Sound union of the osteotomy site was noted in all patient hips in the present study despite some patients having cement in the osteotomy gap. This result is encouraging for surgeons who prefer to use cemented stems with subtrochanteric transverse osteotomy for patients with Crowe IV DDH.

When there is potential fragility in the femur, cemented stems are preferred over uncemented ones because they provide more rotational stability [37]. It is important to note that the surgical procedure itself weakens the femur, especially during the femoral trial step, and cementless stems are associated with a higher risk for intraoperative fractures with an incidence of $0-22 \%[28,33,34]$. Femoral fractures decrease the stability of the standard-length stem, as a result a longer stem with or without additional fixation, such as a cable or plate, is needed. In comparison, intraoperative fractures rarely occur with cemented stems $[8,13-17]$. Should they occur, the cemented stem itself provides good fixation.

To promote union, one study recommends an optimal osteotomy level that depends on the length of the resected femur [24]. Furthermore, the osteotomy ends should be smooth [38] and parallel [14] to maximize the surface contact between the bones. Periosteal stripping should also be kept to a minimum [38]. To reduce the likelihood of cement leakage into the osteotomy gap, we drilled three to five 3-mm holes near the osteotomy sites of the proximal and distal cortical bones for patients included in the present study. The holes allowed the excess cement to leak out rather than deposit into the osteotomy gap during cementing. Another reason was to increase the stability of the cement mantle by the cement column. In addition, it was important to hold both femoral fragments using bone clamps during the process to avoid collecting cement leakage in the osteotomy site. 
There is evidence that the stem bridging the osteotomy site is vital for achieving stability, but the recommended length varies in each study. Ozan et al. [39] proposed that the femoral stem should pass the osteotomy site by at least 4-5 cm, whereas Yang et al. [40] suggested that at least $3 \mathrm{~cm}$ was sufficient. Meanwhile, Kawai et al. [15] proposed that surgeons should insert the standard-length stem to a depth of at least $7 \mathrm{~cm}$ below the osteotomy site. This allows the stem to function as an intramedullary nail, providing stability. Charity et al. [14] concluded that the stem should bypass the osteotomy site by at least twice the diameter of the diaphysis, especially in patients who require more robust fixation, such as in young men. In this study, the average stem length used to bridge the osteotomy site was $97.6 \mathrm{~mm}$ (range, 76.5-106.2 mm), consistent with the data presented in the studies mentioned.

While all the patients in the present study demonstrated significant improvement after surgery, some postoperative complications were also noted. Two patients experienced hip dislocation within 3 months after surgery (Fig. 2). This represents a dislocation rate of $14.3 \%(2 / 14)$, which corresponds with the published dislocation rate of $3.8-15 \%$ in patients with Crowe IV DDH who undergo subtrochanteric osteotomy $[1,7,8,13,16,33]$. Both patients underwent closed reduction, were required to wear a brace, and were put on bed rest for 4 weeks. No dislocations recurred throughout the study. Moreover, while all the osteotomy sites demonstrated good union, one patient showed delayed healing at 18 months postoperatively (Fig. 1). Analysis of her postoperative radiographs revealed a linear hyperdense shadow in the osteotomy gap, which persisted throughout follow-up. Since no bone growth was observed within the shadow, we suspected that the shadow was cement within the osteotomy gap. The piece of cement prevented bone healing and promoted bone resorption, which was represented by the presence of a lowdensity shadow within the cortical bone around the osteotomy site. In the serial radiographs of this patient, new bone formation only occurred at the cortical bone periphery, which prolonged bone union time. No other complications occurred during the study period.

We also analyzed the correlation between osteotomy union time and several other factors, such as the length of the femoral resection, length of limb lengthening, distance of the osteotomy site from the lesser trochanter, and length of the stem to bridge the osteotomy site. The results suggest that there is no statistically significant correlation among these factors. Our data are similar to those presented by Akiyama et al. [16]. In contrast, Kawai et al. [36] demonstrated a significant correlation between the length of bone resection and the incidence of delayed union. However, Kawai et al. [36] divided the resected femoral fragments into two or three longitudinal segments and fixed these to the osteotomy site as autologous bone plates. This difference in technique may be the reason behind the differing results in the two studies.

This study had some limitations. First, there was no control group owing to the retrospective study design. Second, the number of enrolled patients is relatively small, with data obtained from only 14 individuals. However, Crowe IV DDH is relatively uncommon, so obtaining a larger sample from a single institution may be difficult [13]. Third, our follow-up period was limited to a maximum of 70 months. Continued follow-up past 70 months may be beneficial to establish more long-term results associated with this procedure. 


\section{Conclusions}

Cemented stem THA combined with subtrochanteric femoral shortening and transverse derotational osteotomy is safe and effective for the treatment of patients with Crowe IV DDH. The cemented femoral component showed promising mid-term follow up results. The long-term results of this procedure should be evaluated in future studies. The study also confirmed that cement leakage into the osteotomy gap significantly slowed the bone healing process. As such, osteotomy and the cementing process should be performed with great care.

\section{Abbreviations}

DDH developmental dysplasia of the hip

HHS Harris hip score

LLD limb length discrepancy

THA total hip arthroplasty.

\section{Declarations}

\section{Ethics approval and consent to participate}

The research was performed according to the principles of the Declaration of Helsinki and was approved by our institutional ethics review board

The name of the ethics committee: Medical Technology Clinical Application Management Committee of Ningbo No.6 Hospital

The reference number: No L2021023

\section{Consent for publication}

Not applicable

\section{Availability of data and materials}

The datasets generated and analyzed during the current study are not publicly available because the data are not public, but are available from the first author on reasonable request.

\section{Competing interests}


The authors declare that they have no competing interests

\section{Funding}

Not applicable

\section{Author contributions}

Conception and design of study: ZYH, JHZ, ZLD, KT. Performing the operation: ZLD. Acquisition of data: ZYH, KT. Analysis and interpretation of data: ZYH, JL, ZMZ, ZLD, KT. Manuscript drafting: ZYH, KT. Revising the article: All of authors.

\section{Acknowledgements}

We thank Editage for assisting in the preparation of this manuscript

\section{References}

1. Park MS, Kim KH, Jeong WC. Transverse subtrochanteric shortening osteotomy in primary total hip arthroplasty for patients with severe hip developmental dysplasia. J Arthroplasty 2007;22:1031-6.

2. Sugano N, Noble PC, Kamaric E, Salama JK, Ochi T, Tullos HS. The morphology of the femur in developmental dysplasia of the hip. J Bone Joint Surg Br 1998;80:711-9.

3. Watts CD, Abdel MP, Hanssen AD, Pagnano MW. Anatomic Hip Center Decreases Aseptic Loosening Rates After Total Hip Arthroplasty with Cement in Patients with Crowe Type-II Dysplasia: A Concise Follow-up Report at a Mean of Thirty-six Years. J Bone Joint Surg Am 2016;98:910-5.

4. Bicanic G, Delimar D, Delimar M, Pecina M. Influence of the acetabular cup position on hip load during arthroplasty in hip dysplasia. Int Orthop 2009;33:397-402.

5. Lai KA, Shen WJ, Huang LW, Chen MY. Cementless total hip arthroplasty and limb-length equalization in patients with unilateral Crowe type-IV hip dislocation. J Bone Joint Surg Am 2005;87:339-45.

6. Rogers BA, Garbedian S, Kuchinad RA, Backstein D, Safir O, Gross AE. Total hip arthroplasty for adult hip dysplasia. J Bone Joint Surg Am 2012;94:1809-21.

7. Kilicoglu OI, Turker M, Akgul T, Yazicioglu O. Cementless total hip arthroplasty with modified oblique femoral shortening osteotomy in Crowe type IV congenital hip dislocation. J Arthroplasty 2013;28:117-25.

8. Masonis JL, Patel JV, Miu A, Bourne RB, McCalden R, Macdonald SJ, Rorabeck CH. Subtrochanteric shortening and derotational osteotomy in primary total hip arthroplasty for patients with severe hip 
dysplasia: 5-year follow-up. J Arthroplasty 2003;18:68-73.

9. Reikeraas O, Lereim P, Gabor I, Gunderson R, Bjerkreim I. Femoral shortening in total arthroplasty for completely dislocated hips: 3-7 year results in 25 cases. Acta Orthop Scand 1996;67:33-6.

10. Huo MH, Zatorski LE, Keggi KJ. Oblique femoral osteotomy in cementless total hip arthroplasty. Prospective consecutive series with a 3-year minimum follow-up period. J Arthroplasty 1995;10:31927.

11. Hotokebuchi T, Sonohata M, Shigematsu M, Mawatari M. A new device for a V-shaped subtrochanteric osteotomy combined with total hip arthroplasty. J Arthroplasty 2006;21:135-7.

12. Paavilainen T, Hoikka V, Solonen KA. Cementless total replacement for severely dysplastic or dislocated hips. J Bone Joint Surg Br 1990;72:205-11.

13. Howie CR, Ohly NE, Miller B. Cemented total hip arthroplasty with subtrochanteric osteotomy in dysplastic hips. Clin Orthop Relat Res 2010;468:3240-7.

14. Charity JA, Tsiridis E, Sheeraz A, Howell JR, Hubble MJ, Timperley AJ, Gie GA. Treatment of Crowe IV high hip dysplasia with total hip replacement using the Exeter stem and shortening derotational subtrochanteric osteotomy. J Bone Joint Surg Br 2011;93:34-8.

15. Kawai T, Tanaka C, Ikenaga M, Kanoe H. Cemented total hip arthroplasty with transverse subtrochanteric shortening osteotomy for Crowe group IV dislocated hip. J Arthroplasty 2011;26:229-35.

16. Akiyama H, Kawanabe K, Yamamoto K, Kuroda Y, So K, Goto K, Nakamura T. Cemented total hip arthroplasty with subtrochanteric femoral shortening transverse osteotomy for severely dislocated hips: outcome with a 3- to 10-year follow-up period. J Orthop Sci 2011;16:270-7.

17. Oe K, lida H, Nakamura T, Okamoto N, Wada T. Subtrochanteric shortening osteotomy combined with cemented total hip arthroplasty for Crowe group IV hips. Arch Orthop Trauma Surg 2013;133:176370.

18. Li C, Zhang C, Zhang M, Ding Y. Comparison of transverse and modified subtrochanteric femoral shortening osteotomy in total hip arthroplasty for developmental dysplasia of hip: a meta-analysis. BMC Musculoskelet Disord 2014;15:331.

19. Ogawa H, Ito Y, Shinozaki M, Matsumoto K, Shimizu K. Subtrochanteric transverse shortening osteotomy in cementless total hip arthroplasty achieved using a modular stem. Orthopedics 2011;34:170.

20. DeLee JG, Charnley J. Radiological demarcation of cemented sockets in total hip replacement. Clin Orthop Relat Res 1976:20-32.

21. Gruen TA, McNeice GM, Amstutz HC. "Modes of failure" of cemented stem-type femoral components: a radiographic analysis of loosening. Clin Orthop Relat Res 1979:17-27.

22. Johnston RC, Fitzgerald RH, Jr., Harris WH, Poss R, Muller ME, Sledge CB. Clinical and radiographic evaluation of total hip replacement. A standard system of terminology for reporting results. J Bone Joint Surg Am 1990;72:161-8. 
23. Takao M, Ohzono K, Nishii T, Miki H, Nakamura N, Sugano N. Cementless modular total hip arthroplasty with subtrochanteric shortening osteotomy for hips with developmental dysplasia. J Bone Joint Surg Am 2011;93:548-55.

24. Huang ZY, Liu H, Li M, Ling J, Zhang JH, Zeng ZM. Optimal location of subtrochanteric osteotomy in total hip arthroplasty for crowe type IV developmental dysplasia of hip. BMC Musculoskelet Disord 2020;21:210.

25. Linde F, Jensen J. Socket loosening in arthroplasty for congenital dislocation of the hip. Acta Orthop Scand 1988;59:254-7.

26. Togrul E, Ozkan C, Kalaci A, Gulsen M. A new technique of subtrochanteric shortening in total hip replacement for Crowe type 3 to 4 dysplasia of the hip. J Arthroplasty 2010;25:465-70.

27. Muratli KS, Karatosun V, Uzun B, Celik S. Subtrochanteric shortening in total hip arthroplasty: biomechanical comparison of four techniques. J Arthroplasty 2014;29:836-42.

28. Bruce WJ, Rizkallah SM, Kwon YM, Goldberg JA, Walsh WR. A new technique of subtrochanteric shortening in total hip arthroplasty: surgical technique and results of 9 cases. J Arthroplasty 2000;15:617-26.

29. Woolson ST, Harris WH. Complex total hip replacement for dysplastic or hypoplastic hips using miniature or microminiature components. J Bone Joint Surg Am 1983;65:1099-108.

30. Halley DK, Wroblewski BM. Long-term results of low-friction arthroplasty in patients 30 years of age or younger. Clin Orthop Relat Res 1986:43-50.

31. Jasty M, Anderson MJ, Harris WH. Total hip replacement for developmental dysplasia of the hip. Clin Orthop Relat Res 1995:40-5.

32. Sener N, Tozun IR, Asik M. Femoral shortening and cementless arthroplasty in high congenital dislocation of the hip. J Arthroplasty 2002;17:41-8.

33. Zeng WN, Liu JL, Wang FY, Zhang X, Fan HQ, Chen GX, Guo L, Duan XJ, Zhou Q, Yang L. Total hip arthroplasty for patients with Crowe type IV developmental dysplasia of the hip: Ten years results. Int J Surg 2017;42:17-21.

34. Krych AJ, Howard JL, Trousdale RT, Cabanela ME, Berry DJ. Total hip arthroplasty with shortening subtrochanteric osteotomy in Crowe type-IV developmental dysplasia. J Bone Joint Surg Am 2009;91:2213-21.

35. Prins W, Meijer R, Kollen BJ, Verheyen CC, Ettema HB. Excellent results with the cemented Lubinus SP II 130-mm femoral stem at 10 years of follow-up: 932 hips followed for 5-15 years. Acta Orthop 2014;85:276-9.

36. Kawai T, Goto K, Kuroda Y, Matsuda S. Total Hip Arthroplasty Combined With Subtrochanteric Transverse Shortening Osteotomy: Factors Associated With Delayed Union at the Osteotomy Site. J Am Acad Orthop Surg Glob Res Rev 2020;4:e2000056.

37. Takeda Y, Fukunishi S, Nishio S, Fujihara Y, Yoshiya S. Subtrochanteric femoral shortening osteotomy concomitantly performed with revision total hip arthroplasty: A case report. Medicine (Baltimore) 2018;97:e12934. 
38. Wang D, Li LL, Wang HY, Pei FX, Zhou ZK. Long-Term Results of Cementless Total Hip Arthroplasty With Subtrochanteric Shortening Osteotomy in Crowe Type IV Developmental Dysplasia. J Arthroplasty 2017;32:1211-9.

39. Ozan F, Uzun E, Gurbuz K, Koyuncu S, Altay T, Kayali C. Total hip arthroplasty in the developmental dysplasia of the hip using transverse subtrochanteric osteotomy. J Orthop 2016;13:259-63.

40. Yang TC, Chen CF, Tsai SW, Chen WM, Chang MC. Does restoration of hip center with subtrochanteric osteotomy provide preferable outcome for Crowe type III-IV irreducible development dysplasia of the hip? J Chin Med Assoc 2017;80:803-7.

\section{Figures}
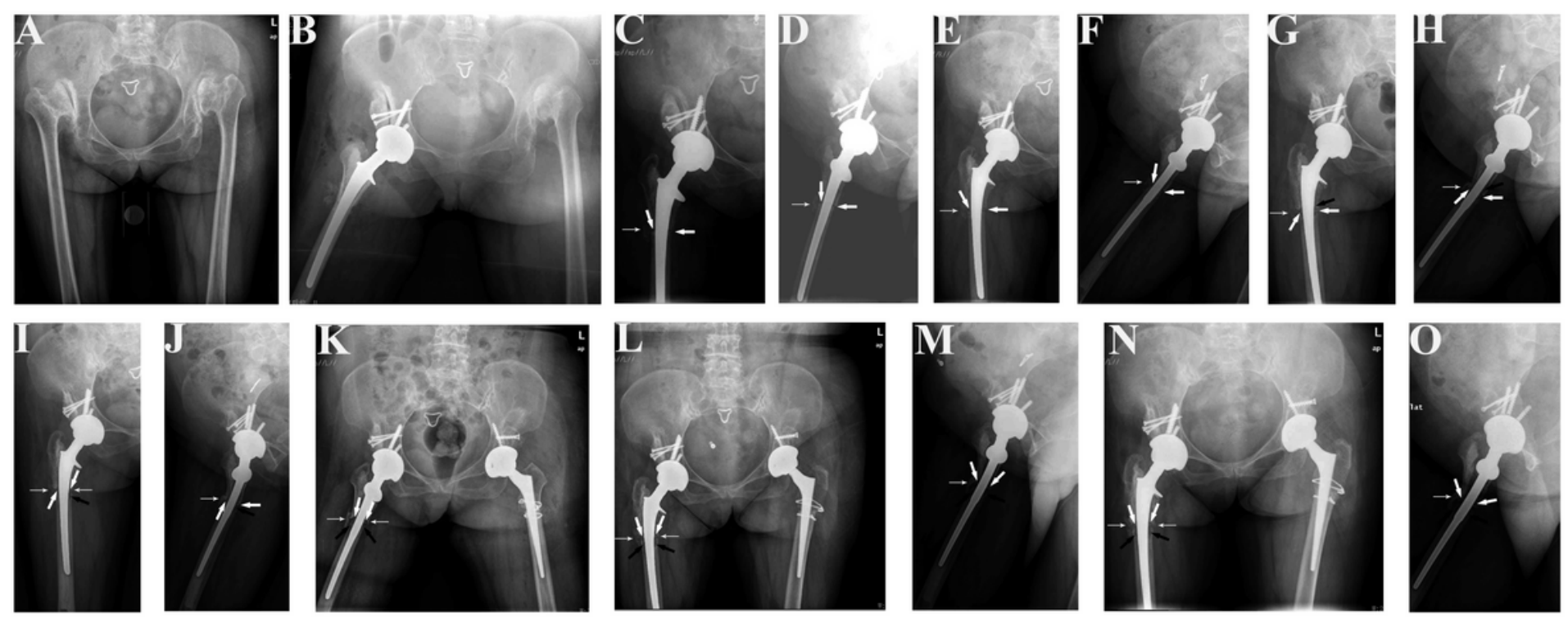

\section{Figure 1}

Radiographs of a 51-year-old woman with bilateral Crowe IV developmental dysplasia of the hip. (A) Preoperative anteroposterior view. (B) Postoperative radiographic image from the first postoperative day. Right total hip arthroplasty was performed with a cemented stem and subtrochanteric femoral shortening derotational transverse osteotomy. $(\mathrm{C}-\mathrm{O})$ Postoperative radiographs showing cement within the osteotomy gap. The radiographs show the bone bridge located at the cortical bone periphery at 3-month follow-up (C-D), 5-month follow-up (E-F), 10-month follow-up (G-H), 17-month follow-up (I-J), 18-month follow-up $(\mathrm{K})$, 21-month follow-up $(\mathrm{L}-\mathrm{M})$, and 31-month follow-up (N-O). From the 10-month follow-up until the last follow-up, bone resorption is noted in the cortical bone around the osteotomy site. At the 18month follow-up, a mature bone bridge is noted around the osteotomy site. The contralateral hip also shows a cementless hip implant. Both hips show autogenous femoral head structural bone grafts for acetabular deficiency. No signs of component loosening are identified. The thick white arrows indicate cement within the osteotomy gap; the thin white arrows indicate the bone bridge around the osteotomy site; and the thick black arrows indicate bone resorption within the cortical bone. 

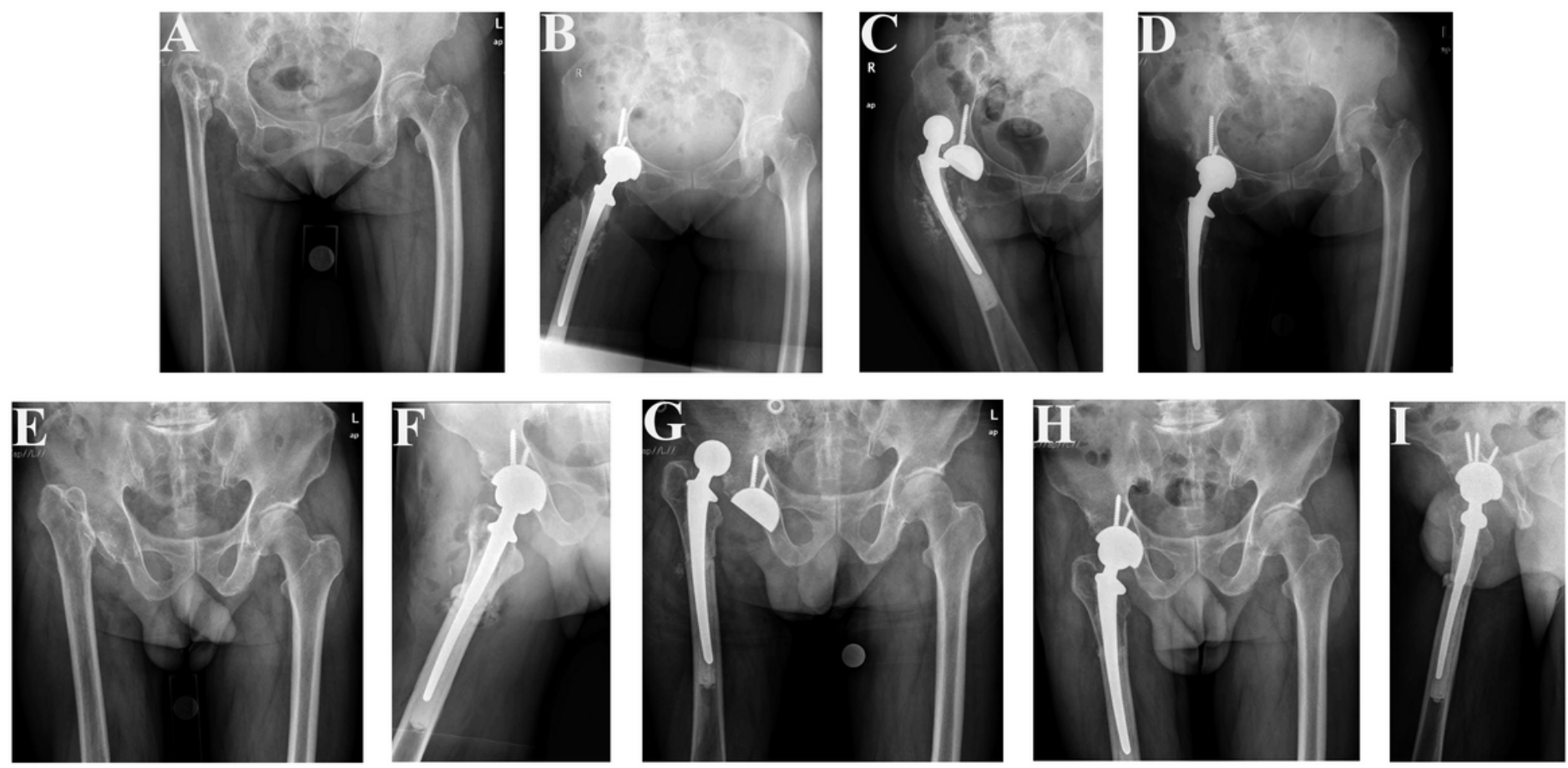

\section{Figure 2}

Radiographs of two patients with Crowe IV developmental dysplasia of the hip. (A, E) Preoperative anteroposterior view of a 73-year-old woman and a 68-year-old man, respectively. (B, F) Postoperative radiographic images on the first postoperative day. Total hip arthroplasty was performed using cemented stem and subtrochanteric femoral shortening derotational transverse osteotomy. (C, G) Postoperative radiographs show hip dislocation during the first and third months after surgery, respectively. $(D, H-I)$ At the 8- and 11-month follow-ups, respectively, the bone union is detected at the osteotomy site. No evidence of loosening is observed. 